\title{
UNA APROXIMACIÓN \\ A LOS ESTUDIOS SOBRE LA \\ Arquidiócesis de Bogotá
}

\author{
Carlos Mario Alzate Montes, O. P. \\ Fabián Leonardo Benavides Silva
}

La obra compilatoria sobre la Arquidiócesis de Bogotá que se presenta a continuación pretende conmemorar los 450 ańos de su historia, relevando la impronta sociocultural, económica y política de esta institución en Colombia, a través de los periodos hispánico, republicano y contemporáneo. Junto a esta celebración, nos asiste el interés fundamental de abordar desde distintas perspectivas disciplinares algunas de las temáticas que no han sido tratadas, deslindando su estudio del tono y del orden meramente cronológico, anecdótico, biográfico y/o hagiográfico imperantes hasta el momento, los cuales han impedido entender con mayor claridad los factores (relaciones con otras instancias sociales o posiciones ideológicas) y las circunstancias (coyunturas o crisis en cualquier orden) que han determinado la labor evangelizadora de esta institución.

Aunque es innegable la importancia de la Arquidiócesis de Bogotá y en general de toda la Iglesia católica en la historia colombiana ${ }^{1}$, es fundamental establecer los acontecimientos, personajes, dinámicas, fuentes, discursos, y en suma, todos los elementos que permitan tanto definir la obra de la Arquidiócesis, como determinar su participación en los diferentes procesos de la vida nacional.

${ }^{1}$ Especialmente si se tiene en cuenta que aún la mayoría de la población colombiana pertenece a ella o por lo menos se identifica con sus preceptos, pese al incremento de diversos movimientos religiosos en Colombia, pues se estima que $70.9 \%$ de sus pobladores se consideran católicos en la actualidad (Beltrán, 2012, p. 209). 
Con el ánimo de identificar con precisión las temáticas y las perspectivas metodológicas en los estudios históricos sobre dicha institución, al tiempo que señalar posibles vacios teóricos al respecto, reseñamos a continuación los trabajos que han gozado de mayor difusión y circulación hasta el momento. Estos trabajos los hemos organizado por temáticas y de acuerdo a la especificidad con el objeto de estudio.

\section{ESTADO DE LA CUESTIÓN}

Entre las obras de carácter específico sobre la Arquidiócesis se destaca Mestizos y Heraldos de Dios (2012) del historiador Juan Fernando Cobo Betancourt que, enfocado en el trabajo administrativo y pastoral del arzobispo de Santafé, fray Luis Zapata de Cárdenas, analiza las implicaciones que tuvo la ordenación de forma masiva de clérigos seculares y entre estos mestizos, con el fin de solventar diversas problemáticas de la labor evangelizadora en el Nuevo Reino de Granada. El debate teológico y jurídico-conceptual suscitado por el ordenamiento de sacerdotes de descendencia no europea, el cual trascendió en los ámbitos pontificio e imperial, es precisamente el objeto de estudio de Cobo, al determinar cómo surgieron las categorías de distinción racial y de qué manera fueron definiendo la identidad de los diferentes grupos étnicos en las colonias españolas.

Luis Carlos Mantilla, O.F.M., en Historia de la Arquidiócesis de Bogotá. Su itinerario evangelizador, 1564-1993 (1994), se propuso -como lo señala el subtítulo- mostrar las circunstancias que han determinado la labor evangelizadora de esta institución desde el momento de su creación hasta el presente. Sin embargo, a tiempo advierte el autor que dicha misión "[...] solo se desarrolla por mediación de formas concretas de comportamiento y de organización humanas"; es decir que a pesar de abordarse el estudio de "una iglesia particular", es inevitable -y aún indispensable- hacer referencia a los hechos que demarcan "[...] el contexto peculiar de dicho itinerario" (1994, p. 1). Siguiendo esa premisa, Mantilla organiza la obra en doce capítulos, en los cuales aborda temáticas como los principios legales y territoriales que rigieron el devenir de la Arquidiócesis, el desarrollo de las estructuras pastorales y del papel de sus ministros, y las contingencias materiales, ideológicas y políticas que tuvo que sortear para difundir su mensaje.

También se puede incluir en esta misma categoría de obras específicas el trabajo de Julio César Orduz (1987), La arquidiócesis de Santafé de Bogotá en 
la Conquista y la Colonia. Si bien resalta la ausencia de fuentes y citas -quizá por un interés más descriptivo que analítico-, de todas maneras la obra organiza de forma cronológica datos muy importantes sobre los albores de la Arquidiócesis santafereña, separando las principales incidencias según los distintos periodos arzobispales, desde fray Juan de Los Barrios en 1554 hasta la larga vacancia de la silla metropolitana entre 1817 y 1827.

Asimismo, aunque se trata de una referencia más general sobre la historia de la Iglesia, vale mencionar aquí el importante trabajo adelantado por Juan Manuel Pacheco, S. J. y Roberto María Tisnés, C.M.F., para la Historia Extensa de Colombia (1986). En efecto, el volumen XIII de esta colección subtitulado "Historia Eclesiástica", está dividido en cuatro tomos (los primeros tres elaborados por Pacheco y el cuarto por Tisnés) organizados de la siguiente manera:

El tomo I discurre sobre la introducción del cristianismo en el Nuevo Reino de Granada durante el siglo XVI, es decir, el proceso de evangelización de los indígenas y el papel de la religión y de la Iglesia católica en la configuración de la sociedad neogranadina. En el tomo II el autor estudia el afianzamiento de la Iglesia durante el siglo XVII, especialmente las implicaciones que tuvo el Concilio de Trento en las políticas eclesiásticas y el trabajo pastoral del clero. En el tomo III (compuesto por dos libros), Pacheco examina el devenir de la Iglesia neogranadina en el siglo XVIII, destacando el influjo de sus propios cambios institucionales sobre las ideas ilustradas y las políticas borbónicas de centralización administrativa (que dieron lugar por ejemplo, a la creación del Virreinato en 1739). También se percibe la agitación política al final de la centuria y el malestar en contra de la Corona española, fenómenos que propiciaron el estallido de la emancipación de sus colonias.

Precisamente, en el tomo IV Tisnés estudia la situación de la Arquidiócesis de Santafé en el proceso de Independencia de la actual Colombia, por ese entonces nominalmente a la cabeza del arzobispo Juan Bautista Sacristán, pero administrada en realidad por el Cabildo Catedralicio con gran influjo del clero regular. Aunque la crítica historiográfica más reciente cuestiona la pertinencia de los enfoques y los métodos de este tipo de obras monumentales, quizá su valor reside en los documentos inéditos que referencian, así como en el inventario -a manera de estado del arte- de las publicaciones que citan. Para nuestro objeto de estudio resultan de especial interés la gran cantidad de precisiones y datos históricos, especialmente para el periodo hispánico, momento en el cual jurisdiccionalmente la Arquidiócesis abarcaba más de la tercera parte del territorio neogranadino. 
Dentro de esta categoría de obras específicas existe un grupo de publicaciones que nos permitimos referir con cierto detalle. Se trata de los artículos y disertaciones publicadas en el Boletín de Historia y Antigüedades de la Academia Colombiana de Historia (gran parte de ellas elaboradas por religiosos), entre los cuales se encuentran varios estudios sobre la Arquidiócesis de Bogotá que aportan datos y referencias documentales y bibliográficas que pueden ser de interés y utilidad para los investigadores. Entre ellos destacamos el escrito de José Marroquín, "Origen y desarrollo de la jerarquía eclesiástica en Santafé de Bogotá" (1938), que es una memoria sobre la organización jerárquica de la Iglesia católica desde la fundación del Nuevo Reino de Granada hasta la llegada de fray Martín de Calatayud y el nombramiento de fray Juan de Los Barrios como nuevo obispo de Santa Marta y posterior arzobispo de Santafé2.

Similares descripciones realiza monseñor José Restrepo Posada en sus trabajos "Arquidiócesis de Bogotá" (1950) y "El sínodo diocesano de 1556" (1956), al centrarse en los primeros esfuerzos por organizar y normalizar la labor pastoral y administrativa de la nueva arquidiócesis. A Restrepo se debe también "La primitiva Catedral de Santafé" (1943), crónica en la cual examina los episodios de la historia nacional que impulsaron los cambios arquitectónicos de la Catedral desde su primera edificación en 1553, su reconstrucción en 1572 y la ampliación finalizada en 1823. Finalmente, este autor en "El capítulo metropolitano y el 20 de julio" (1960), determina el grado de participación de la Arquidiócesis de Santafé en los sucesos emancipatorios de 1810.

En el Boletín de Historia y Antigüedades también se publicó "Consagraciones episcopales hechas en Bogotá” (1948) de Pedro María Ibánez, un escrito de singular relevancia, puesto que el autor hace un diagnóstico de los documentos

${ }^{2}$ Sobre este mismo tema escribió monseñor José Vicente Castro Silva el artículo "Del gobierno eclesiástico de Santafé de Bogotá" (1963), proporcionando un panorama general de la llegada de los primeros religiosos, la normatividad que los regía, su asentamiento y posterior organización jerárquica en el Nuevo Reino a través de la creación de la Arquidiócesis. Aunque con una perspectiva más amplia territorialmente hablando, monseñor Mario Germán Romero reconstruyó en el escrito "El teatro eclesiástico de la primitiva iglesia de las Indias Occidentales" (1963), el estado de la Iglesia en América a partir del relato realizado por el cronista Gil González en 1643. Tomando datos de la misma crónica, el autor expone las características del primer prelado que arribó a las Indias, así como los aspectos más destacados de cada generación de estos religiosos, a saber: eclesiásticos elegidos por Carlos V, la llegada de los frailes mendicantes y los esfuerzos por organizar al clero secular. 


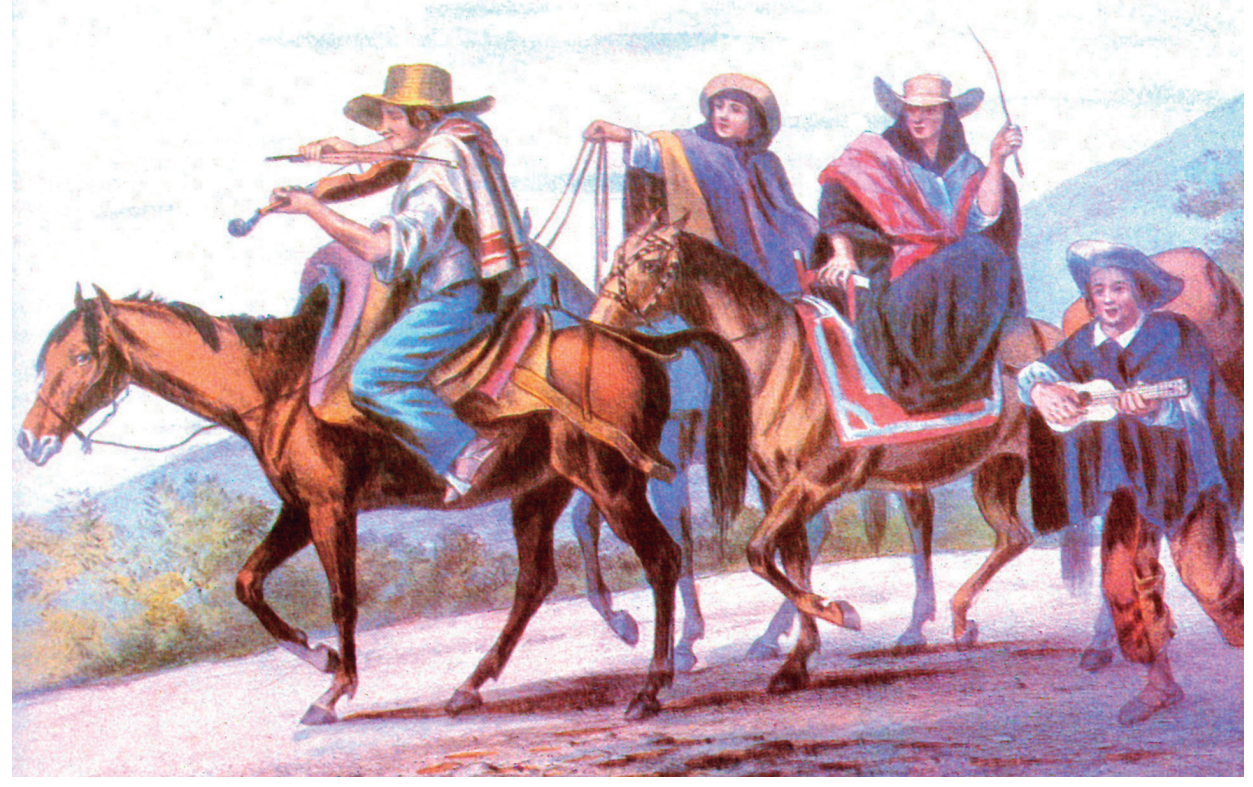

Figura 1. Ramón Torres Méndez. (1809-1885). "Romería a Chiquinquirá". En: Efraín Sánchez Cabra. (1987). Ramón Torres Méndez Pintor de la Nueva Granada. 1809-1885. Bogotá: Fondo Cultural Cafetero.

de las consagraciones episcopales que se perdieron en el incendio del Archivo Arzobispal el 9 de abril de 1948.

Por último, monseñor Germán Pinilla en el artículo "La presencia de la Arquidiócesis de Bogotá en un colegio liberal: el Colegio Nacional de San Bartolomé, 1941-1951” (2011), a partir de la contextualización histórica de este plantel educativo, analiza los cambios internos de la Arquidiócesis de Bogotá en dos coyunturas específicas. La primera de estas dada con la sanción de la Constitución de 1886 y el Concordato de 1887, documentos que concedieron a la sede metropolitana un escenario privilegiado para el desarrollo de su labor pastoral; y la segunda, en el marco de las políticas de los gobiernos liberales a partir de 1930, que pretendieron modificar esa misma legislación con el fin de direccionar el Estado hacia una posición laica.

Una segunda tipología de obras corresponde a aquellas que abordan un amplio conjunto de eventos históricos sobre la historia de la Iglesia en Colombia, en las cuales las referencias sobre la Arquidiócesis son indirec- 
tas, pues asumen a la institución eclesiástica como una unidad, sin tener en cuenta las particularidades de cada una de sus jurisdicciones. Así, Humberto Bronx expone en Historia Moderna de la Iglesia Colombiana (1992) el acaecer histórico de la institución eclesiástica en el país. El autor presenta esquemáticamente la forma en que la Iglesia católica, en tanto agente político, social y cultural, tuvo participación en los momentos determinantes de la historia de la nación (desde la Conquista hasta la contemporaneidad). En todo este recuento se deja entrever el papel de la Arquidiócesis de Bogotá como sede metropolitana o cabeza de la Iglesia, aunque sin precisar las singularidades de su labor sociocultural, económica o política.

En la obra de Michael J. Larosa, De la Derecha a la Izquierda: Historia de la Iglesia Católica en Colombia, 1930-1980 (2000), compuesta por dos tomos, se analizan tres eventos en los que intervino activamente la Iglesia católica en cabeza de la sede primada. En primer lugar, Larosa muestra el apoyo de la Institución en la conformación de grupos de campesinos y trabajadores en respuesta a la crisis económica de las primeras décadas del siglo XX. En segundo lugar ilustra el caso de Camilo Torres a manera de ejemplo de la catolicidad radical en el país, que buscaba alternativas a distintas realidades sociales. El tercer evento revisado es la conferencia de Medellín de 1968, reunión que pretendió crear un itinerario de políticas sociales de la Iglesia, enfocando su trabajo pastoral en la transformación de Latinoamérica a la luz del Concilio Vaticano II. A través de la disertación sobre el desarrollo y la interacción de la Iglesia con las políticas sociales del país, Larosa aporta nuevas perspectivas de análisis a la historia eclesiástica en Colombia. Con el examen de las divisiones en el interior de la institución, se determina la influencia de la misma en asuntos políticos durante los primeros treinta años del siglo XX y la manera en que estas segmentaciones de tipo político en el órgano ministerial, impidieron la consolidación de una voz unánime que expresara el sentir de todos los religiosos en tal periodo.

En Historia General de la Iglesia en América Latina (1981), trabajo liderado por la Comisión para el Estudio de la Historia de la Iglesia en América Latina y el Caribe (CEHILA), el tomo siete de la colección, coordinado por Rodolfo Ramón de Roux, se ocupa de los casos de Colombia y Venezuela. En los trabajos recopilados sobre Colombia escritos por Juan Manuel Pacheco. S.J., Fernán González y Rodolfo Ramón de Roux, se encuentra un panorama general de la historia de la Arquidiócesis santafereña y de sus diferentes actores, destacándose la labor evangelizadora entre la población y la organización de la institución a lo largo de cuatro siglos. 

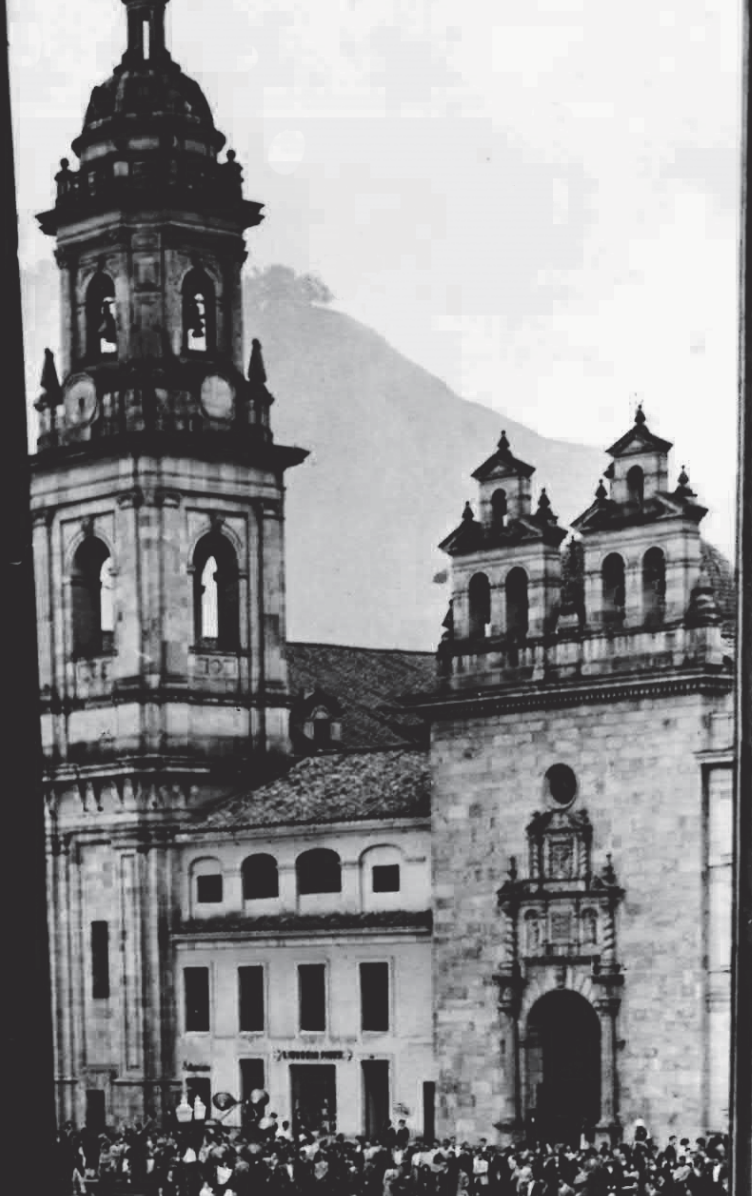

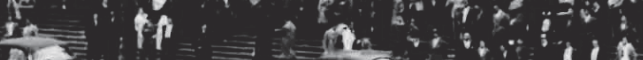

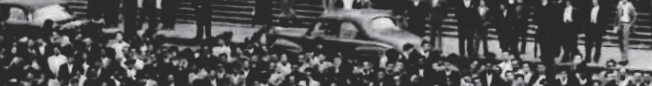

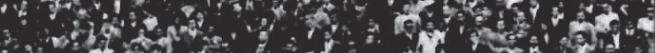
(4)

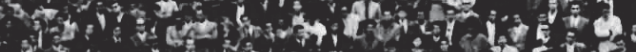

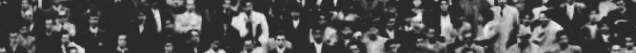

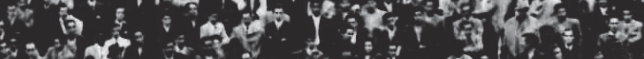

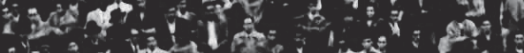
"N

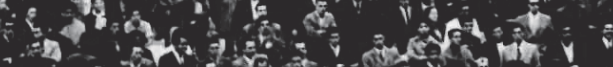

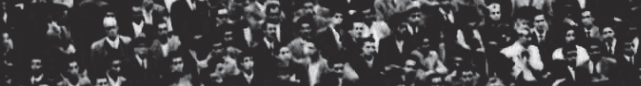
(5.) ${ }^{2}=0$

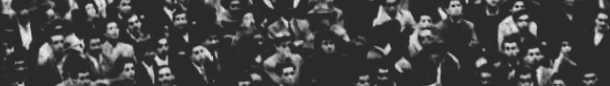
$\therefore$ A 3.

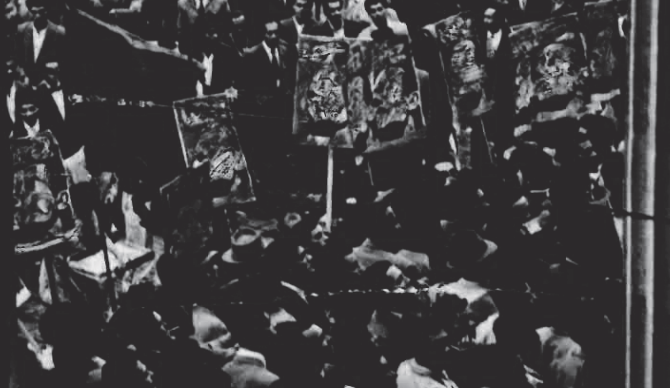


En el primer capítulo de la obra compilatoria titulado "La evangelización en Colombia”, Juan Manuel Pacheco., S. J. examina el proceso de adoctrinamiento de indígenas vivido en el Nuevo Reino de Granada desde el arribo de Gonzalo Jiménez de Quesada en 1537 a la sabana de Bogotá, hasta la configuración de la Junta Suprema de Gobierno en 1810, en cuyo periodo se releva la labor arquidiocesana como centro del gobierno eclesiástico y su relación con las demás órdenes religiosas. En el segundo título "La organización de la Iglesia en Colombia", Pacheco analiza la estructuración jerárquica de la Iglesia católica en el Nuevo Reino de Granada, especialmente de la Arquidiócesis de Santafé y sus diócesis sufragáneas. Además de presentar los cambios organizacionales que respondían a su vez a las cambiantes políticas de evangelización así como a la relación del clero secular y regular con el poder civil, el autor expone la normativa sancionada en las constituciones sinodales referentes a la administración de los sacramentos y a la disciplina de los clérigos en este territorio.

En el tercer apartado del mismo autor "La vida cotidiana de la cristiandad en Colombia", se estudia el papel desempeñado por el Cabildo Eclesiástico en la sociedad colonial del Nuevo Reino, que por un lado, constituyó el cuerpo consejero del arzobispo, y por otro, asumió el gobierno de la diócesis durante las frecuentes y largas vacantes en la silla arzobispal. En el capítulo se enuncia también la influencia de las políticas de gobierno establecidas por los arzobispos Francisco Cossío y Otero y fray Francisco del Rincón, quienes reunieron bajo su administración el poder civil y eclesiástico en las primeras décadas del siglo XVIII.

En la siguiente sección se establecen los procesos independentistas vividos en los actuales territorios de Colombia y Venezuela. Fernán González analiza en el capítulo cuarto, "La Iglesia ante la Emancipación en Colombia”, el papel desempeñado por esta institución en el proceso de transición política y las implicaciones de las ideas liberales en la sociedad y en la propia Iglesia de la naciente nación. En el capítulo quinto, "La Iglesia en la formación del Estado Colombiano (1830-1850)”, González evalúa asimismo la participación política de la Iglesia católica en la consolidación del Estado hacia mediados del siglo XIX. En el sexto acápite, titulado "La reorganización de la Iglesia ante el Estado liberal y su crisis (1850-1886)", este mismo autor se centra en el conflicto suscitado a mediados del siglo XIX, por la injerencia de la Iglesia católica en asuntos políticos. González expone el panorama general del país en los planos político, económico y social, enfocándose en la resistencia asumida por el clero con relación a las políticas económicas emitidas por los gobiernos liberales de este periodo. 
Rodolfo Ramón de Roux determina en el séptimo capítulo, "La Iglesia Colombiana en el periodo 1930-1962", el trabajo pastoral del clero secular regulado por la Arquidiócesis de Bogotá, en respuesta a la llamada problemática socio-pastoral del país. El autor en mención determina el grado de influencia de la Iglesia en la organización de los trabajadores y campesinos en cumplimiento a una agenda en políticas sociales, las cuales habían sido pactadas en las Conferencias Episcopales con el fin de dinamizar la participación de la Iglesia en la vida social de la nación. En el último título "La Iglesia desde el Concilio Vaticano II y Medellín", De Roux estudia las implicaciones sociales del Concilio Vaticano II y del Congreso Eucarístico Internacional de 1968. Por ejemplo, con la introducción del "espíritu conciliar" fundamentado en el diálogo y la apertura al mundo, el Concilio significó el fin de una época y abrió un periodo de mayor libertad de expresión y de disensión dentro de la Iglesia.

Existe una tercera categoría de obras -mucho más prolífica que las anteriores- la cual corresponde a aquellos trabajos en los que el eje de la narración y del análisis (cuando lo hay), se articula a partir de la vida de uno o varios arzobispos. Se trata de biografías y selecciones documentales que, desde luego, contienen muchas de las páginas más importantes de la historia de la Arquidiócesis. En esta categoría sobresale el monumental trabajo de José Restrepo Posada realizado entre 1961 y 1971, titulado Arquidiócesis de Bogotá. Este trabajo está dividido en cuatro tomos así: el tomo I (1961) presenta los datos biográficos de todos los arzobispos de Santafé correspondientes al periodo colonial (35 biografías hasta Isidoro Domínguez, arzobispo electo en 1817, pero que nunca se presentó a su cargo). El tomo II (1963) se ocupa de las biografías de los arzobispos Francisco Caycedo y Flórez (1827-1832), Manuel José Mosquera (1835-1853) y Antonio Herrán y Zaldúa (1855-1868). El tomo III (1966) describe las vidas y los periodos de Vicente Arbeláez (1868-1884), José Telésforo Paúl (1885-1889) e Ignacio León Velasco (1889-1891)3. Finalmente el tomo IV (1971) titulado Cabildo Eclesiástico, recoge los esbozos biográficos de 315 canónigos o capitulares, que en calidad de dignatarios oficiaron como consejeros de los arzobispos

\footnotetext{
${ }^{3}$ Cabe señalar que mientras en el tomo I Restrepo recopila los datos biográficos de 35 prelados, en los tomos II y III apenas muestra las vidas de tres ministros en cada uno. Esto se debe a que en estos volúmenes el autor se explaya mucho más en la historia decimonónica, quizá por tratarse de un periodo de profundas transformaciones y conflictos para la institución eclesiástica.
} 
y en muchas ocasiones, asumieron funciones directivas en el gobierno de la Arquidiócesis durante los periodos de sede vacante . $^{4}$

Otra obra del tipo de las biografías, aunque de carácter más específico, es la de Martha Isabel Márquez, titulada Monseñor Bernardo Herrera Restrepo en la vida política colombiana (1986). Esta es una crónica orientada más hacia la influencia política de este prelado que gobernó en el periodo comprendido entre 1891 y 1928 y al cual se le atribuye cierto patrocinio hacia los gobiernos de la llamada República Conservadora.

Un ejercicio similar al anterior lo realiza Orduz en su libro Monseñor Ismael Perdomo y su tiempo: biografía documentada del insigne Arzobispo de Bogotá (1984). Aunque en algunos apartes adopta un tono novelesco, la obra introduce varias cartas y documentos completos que pueden ser fuentes importantes para la investigación.

Monseñor Guillermo Agudelo en su escrito Medio siglo en la historia eclesiástica colombiana. 1928-1984: ensayo sobre los cuatro Arzobispos que han dejado huella profunda en la agitada historia contemporánea de Colombia (1986), presenta las biografías y los principales acontecimientos ocurridos durante los periodos de los arzobispos Ismael Perdomo Borrero (1928-1950), Crisanto Luque (1950-1959), Luis Concha Córdoba (1959-1967) y Aníbal Muñoz Duque (1972-1984). También de Agudelo se menciona la obra Los arzobispos de Bogotá que han marcado nuestra historia, 1564-2010 (2010), compuesta por los perfiles biográficos de los ministros que han ocupado la silla arzobispal, destacando su relación con las autoridades civiles. Este texto que fue editado con ocasión de la conmemoración del bicentenario de la Independencia de Colombia, incluye también la descripción de algunos aspectos sobre la organización de la Arquidiócesis, así como la narración de varios hechos que determinaron esa configuración.

Pedro María Ibáńez es el autor de los ensayos "Inhumación en la Catedral de los restos de tres arzobispos" (1957) y "Provisores del arzobispado" (1967). En el primer escrito, con motivo de la inhumación de los restos de los arzobispos fray Juan de Dios Aguinao, O. P. (1661-1678), fray Agustín Camacho y

${ }^{4}$ Otros estudios de Restrepo Posada publicados en el Boletín de Historia y Antigüedades son: "El capelo cardenalicio y los arzobispos de Bogotá" (1954), en el que expone las vicisitudes vividas por los arzobispos Antonio Caballero y Góngora y Manuel José Mosquera en sus frustradas designaciones como cardenales; "Arzobispos y obispos de Bogotá, bogotanos que han llegado a la Mitra" (1967), un listado biográfico de veinte religiosos nacidos en la ciudad que estuvieron a cargo de la Arquidiócesis; y por último, "Partidas de bautismo de los arzobispos de Bogotá" (1970), una transcripción de más de quince partidas de bautismo de varios prelados, entre ellos Fernando Arias de Ugarte (1618-1625) y Aníbal Muñoz Duque (1972-1984). 


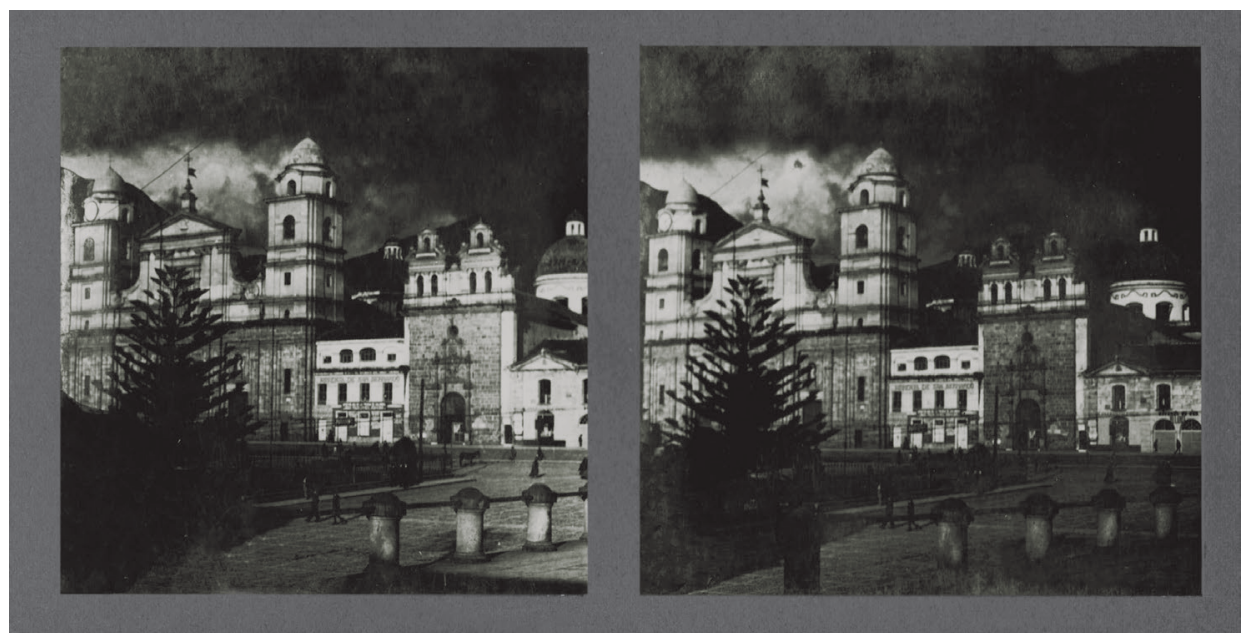

Figura 3. Anónimo. (1910) Fotografía estereoscópica Plaza de Bolivar. Bogotá: Bolívar Old Prints.

Rojas, O. P. (1771-1774) y fray Fernando del Portillo y Torres, O. P. (17981804), efectuada el 5 de octubre de 1957 en la basílica Primada, Ibáńez hace una semblanza biográfica y pastoral de estos tres religiosos. En el segundo texto el autor describe la función del cargo del provisor en la Arquidiócesis, señalando algunos de los aportes de dichos jueces en el gobierno eclesiástico durante el amplio periodo comprendido entre 1553 y 1959, es decir, desde fray Juan de Los Barrios (1564-1569) hasta monseñor Luis Concha Córdoba (1959-1972)5.

Con respecto a las compilaciones documentales podemos mencionar Documentos para la biografía e historia del Episcopado del Ilustrísimo Señor D. Manuel José Mosquera (1858). Esta gran obra está dividida en tres tomos de más de 700 páginas cada uno y recopila documentación de toda índole. Se incluyen disertaciones completas, estatutos, cartas, informes, mensajes, reclamaciones, oficios, leyes, etc. Para dimensionar la funcionalidad de esta documentación, vale la pena recordar que históricamente le correspondió al arzobispo Mosquera la defensa de la Iglesia del embate de las reformas liberales de José Hilario López en 1849, proceso que culminó con el destierro del prelado en 1852.

${ }^{5}$ En su libro Compartir los hallazgos. Páginas de pequeña historia de Colombia (2013) Luis Carlos Mantilla incluye un listado de los artículos publicados en el Boletín de Historia y Antigüedades que han abordado las biografías de los arzobispos de Bogotá desde la erección de 
Debemos referenciar los acuciosos trabajos de Luis Carlos Mantilla titulados Don Bartolomé Lobo Guerrero: inquisidor y tercer arzobispo de Santafé de Bogotá, 1599-1609 (1996); Mitra y sable: correspondencia del Arzobispo Manuel José Mosquera con su hermano el General Tomás Cipriano, 1817-1853 (2004) y Profeta en su tierra: Hernando Arias de Ugarte V Arzobispo de Santafé de Bogotá. Su correspondencia en el Archivo General de Indias de Sevilla (2008). Además de las trascripciones documentales, estas obras introducen los bosquejos biográficos

esta institución hasta la actualidad. Los trabajos relacionados por el autor además de recopilar datos cronológicos, dan cuenta de los cambios normativos e ideológicos de la Iglesia a lo largo de su historia en el país. Los autores y los títulos de estos artículos son: Pedro María Ibánez, "Cuadro Sinóptico de los Arzobispos de Bogotá, desde la creación de la silla. 1553 a 1905" (1905); Diego Mendoza, "Verdadera relación de la sucesión de los ilustrísimos señores arzobispos de esta metrópoli, año de 1764" (1910); Luis Augusto Cuervo, "Nota sobre el arzobispo Hernando Arias de Ugarte" (1922); Arturo Quijano, "El arzobispo prócer [don Fernando Caycedo y Flórez]" (1932); José Restrepo Posada, "Los restos del Ilustrísimo señor Mosquera" (1935); Luis Augusto Cuervo, “El arzobispo Manuel José Mosquera” (1936); Luis Martínez Delgado, "El arzobispo Mosquera” (1936); Ernesto Restrepo Tirado, "Muerte del [arzobispo] Fray Bernardino de Almansa” (1938); Luis Cuervo Márquez, "El arzobispo Manuel José Mosquera" (1942); Daniel Ortega Ricaurte, "Elogio del arzobispo Fernando Caycedo y Flórez" (1945); José Restrepo Posada, "Don Isidoro Domínguez, arzobispo de Santafé” (1951); Horacio Rodríguez Plata, "El arzobispo Mosquera como hombre de Estado" (1953); Jaime Hincapié, "Elogio del arzobispo Mosquera" (1953); José Restrepo Posada, "Elogio del arzobispo Mosquera" (1953); José Restrepo Posada, "Homenaje al señor arzobispo Mosquera" (1954); Marco Tulio Vargas, "Don Fernando del Portillo y Torres, arzobispo de Santafé, 1797-1803" (1955); Bernardo J. Caicedo, "Obra e imagen del arzobispo-prócer [don Fernando Caycedo y Flórez]” (1956); Mario Germán Romero, "El arzobispo Vicente Arbeláez y el II Concilio Provincial Neo-Granadino" (1956); José Alejandro Bermúdez, "Los tunjos de oro del arzobispo Zapata de Cárdenas" (1957); Alfonso Zawadski, "El arzobispo Juan Bautista Sacristán” (1959); Enrique Otero de Acosta, "Sucesión de prelados y jueces: seculares del Nuevo Reino de Granada" (1960); Luis Concha, "Un alegato judicial del arzobispo Mosquera” (1970); J. Restrepo Posada, "El ilustrísimo señor don Fray Luis Zapata de Cárdenas" (1974); Terrence B. Horgan, "Carta del arzobispo de Nueva York al de Bogotá, Manuel José Mosquera" (1979); Roberto M. Tisnés, "El arzobispo Monseñor Vicente Arbeláez, 1822-1884” (1984); Manuel Briceño Jáuregui, "El arzobispo don Bernardino de Almansa, una personalidad discutida" (1985); Armando Gómez Latorre, "Semblanza histórica del arzobispo Baltasar Jaime Martínez" (1994); Josep-Ignasi Saranyana, "Estudio histórico-doctrinal del catecismo de Fray Luis Zapata de Cárdenas, 1576" (1994); Guillermo Agudelo, "Visión general de los arzobispos de Santafé de Bogotá desde la Colonia hasta nuestros días" (1995); Jaime Hincapié, "Bicentenario de los hermanos Manuel José y Manuel María Mosquera" (1999); Luis Carlos Mantilla, "Relaciones entre el arzobispo Manuel Mosquera y su hermano el General Tomás Cipriano" (2000); y Mario Germán Romero, “Monseñor Manuel José Mosquera” (2000). 


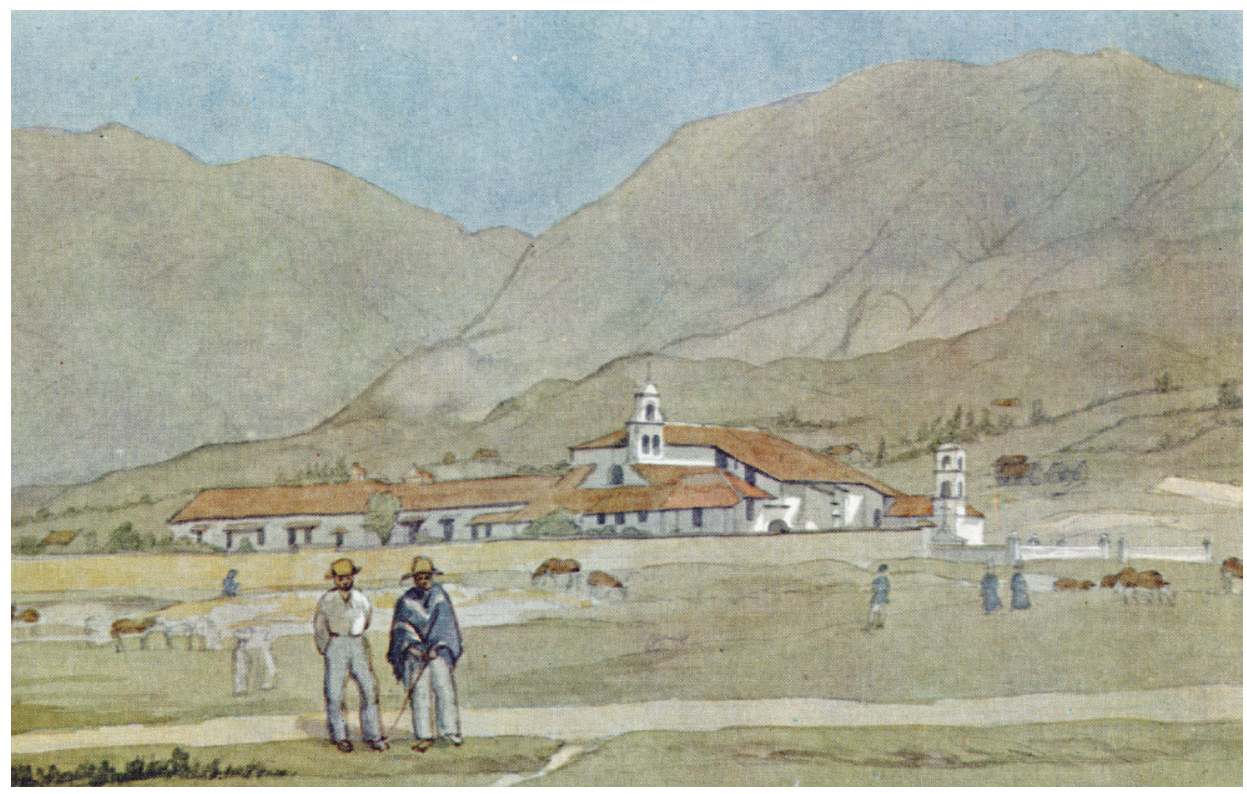

Figura 4. Edward Mark Walhouse. (1850) Recoleta de San Diego. En: Germán Botero. (Comp.), (1976) Acuarelas de Mark. 1843-1856. Un testimonio pictórico de la Nueva Granada. Bogotá: Banco de la Republica, Litografía el Arco. p. 56.

de cada uno de los personajes. Particularmente, la documentación en los trabajos sobre los arzobispos Lobo Guerrero y Arias de Ugarte (1618-1625), recoge temáticas como la evangelización, la construcción de iglesias, la fundación de doctrinas y las relaciones con las autoridades civiles y las comunidades religiosas.

Finalmente, una cuarta categoría de obras corresponde a los estudios sobre la arquitectura y el arte de la Arquidiócesis. Entre estos trabajos cabe destacar el realizado por monseñor Juan Miguel Huertas, El tesoro de la Catedral de Santafé de Bogotá (1995), obra que expone un catálogo de imágenes de los objetos elaborados por maestros artesanos en distintos materiales (oro, plata, cobre y plomo), que a su vez corresponden a diferentes periodos históricos y cuyo valor patrimonial es invaluable. Entre los elementos custodiados por la Catedral Primada sobresalen custodias, cálices, cruces, lavabos, atriles, báculos, vinajeras, broches y alfileres, entre otros. El autor llama la atención sobre el gran significado histórico de los elementos conservados por la Iglesia metropolitana de Santafé, conservándose por ejemplo, el cáliz de plomo utilizado por fray Domingo de las Casas, O. P. en la primera misa celebrada tras la fundación de la ciudad el 6 de agosto de 1538. 
Recientemente y con motivo del jubileo que también nos convocó para la realización del presente trabajo, se editó el libro Arte Sacro (2012) que expone el acervo de obras de arte albergadas en la Arquidiócesis de Bogotá, incluida la Catedral Primada, el Palacio Cardenalicio y otras parroquias de la capital y de municipios cercanos. Como se asevera en el texto, la colección es una muestra representativa de la cultura visual colonial y se encuentra acompañada por un estudio de Jaime Borja que contextualiza cada imagen, determinando el sentido de las obras y su función en la labor evangelizadora en el Nuevo Reino de Granada.

Otra obra que celebra el aniversario 450 de la Arquidiócesis es Iglesias Coloniales, Conventos y Ermitas. Santafé, Arquidiócesis de Bogotá. 450 años (2013), dirigida por el padre Sahamuel Ortiz. El estudio recopila la historia de las iglesias ubicadas en la ciudad. El libro dividido en seis capítulos, examina en su orden: las iglesias parroquiales, los templos de los conventos de hombres y de mujeres, las iglesias asociadas a hospitales, colegios, hospicios y sitios de recogimiento, las ermitas de cultos particulares y por último, las capillas doctrineras que se ubicaban al oriente del río Bogotá, las cuales fungían como centros de evangelización para los indígenas. En cada caso, el análisis se enfoca en la descripción de los edificios y sus objetos de culto, así como en la importancia para la sociedad capitalina de la actividad pastoral que tuvo lugar en cada una de ellas.

\section{BALANCE}

Desde el punto de vista historiográfico, la historia de la Arquidiócesis de Bogotá casi siempre ha sido abordada desde o a través de la historia de sus prelados. En efecto, al revisar los catálogos o bases de datos de las principales bibliotecas, se echan sensiblemente de menos obras o estudios de carácter específico sobre esta institución. En contraste, son más o menos abundantes las biografías, epistolarios y selecciones documentales sobre algunos de los arzobispos más connotados. Aunque en este segundo grupo de obras se encuentran piezas de diversa índole en cuanto a su finalidad y métodos, es innegable que existe en ellas una riqueza de información que aún está por utilizarse y verse articulada a estudios de más largo y particular aliento.

Desde luego hay que destacar también las referencias efectuadas sobre la Arquidiócesis bogotana en la historiografía nacional, aunque consideramos que su especificidad se diluye en las grandes líneas de los estudios históricos sobre la Iglesia en Colombia y en América Latina, en la historia de Colombia y de Bogotá y en general, en las historias de las grandes épocas, los procesos y los acontecimientos. 
Finalmente, es necesario llamar la atención sobre la falta de investigaciones que además de la historia, estudien a la sede metropolitana desde otras disciplinas de las ciencias sociales y humanas con el fin de profundizar en las temáticas que ya han sido abordadas y naturalmente, que inauguren también el análisis de aquellas que no.

\section{REFERENCIAS}

Agudelo, G. (1986). Medio siglo en la historia eclesiástica colombiana. 19281984: ensayo sobre los cuatro Arzobispos que han dejado huella profunda en la agitada historia contemporánea de Colombia. Bogotá: Ediciones Verdad y Vida.

Anónimo. (1858). Documentos para la biografía e historia del Episcopado del Ilustrísimo Señor D. Manuel José Mosquera. Paris: A. Le Clere.

Beltrán, W. (2012, enero-junio). Descripción cuantitativa de la pluralización religiosa en Colombia. Universitas Humanistica, (73), 201-237.

Bronx, H. (1992). Historia Moderna de la Iglesia Colombiana. Medellín: Argemiro Salazar.

Castro, J. (1963). Del gobierno eclesiástico de Santafé de Bogotá. Boletín de Historia y Antigüedades, 50, 349-437.

Cobo. J. F. (2012). Mestizos y Heraldos de Dios. La ordenación de sacerdotes descendientes de españoles e indígenas en el Nuevo Reino de Granada y la racialización de la diferencia. 1537-1590. Bogotá: ICANH.

De Roux, R. (Coord.). (1981). Historia General de la Iglesia en América Latina. VII. Colombia y Venezuela. Salamanca: Ediciones Sígueme S. A.

Huertas, J. (1995). El tesoro de la Catedral de Santafé de Bogotá. Bogotá: Amazonas editores.

Ibáñez, P. (1948). Consagraciones episcopales hechas en Bogotá. Boletín de Historia y Antigüedades, 35, 360-376.

Ibáńez, P. (1957). Inhumación en la Catedral de los restos de tres arzobispos (los dominicos Fray Juan de Arguinao, Fray Agustín Camacho y Rojas y Fray Fernando del Portillo). Boletín de Historia y Antigüedades, 44, 539-549.

Ibáñez, P. (1967). Provisores del arzobispado. Boletín de Historia y Antigüedades, 54, 71-100.

Larosa, M. (2000). De la Derecha a la Izquierda: Historia de la Iglesia Católica en Colombia 1930-1980. Bogotá: Fundación para la promoción de la investigación y la tecnología. 
Mantilla, L. C. (1994). Historia de la Arquidiócesis de Bogotá. Su itinerario evangelizador, 1564-1993. Bogotá: Arquidiócesis de Bogotá.

Mantilla, L. C. (1996). Don Bartolomé Lobo Guerrero: inquisidor y tercer arzobispo de Santafé de Bogotá, (1599-1609). Bogotá: Academia Colombiana de Historia.

Mantilla, L. C. (Ed.). (2004). Mitra y sable: correspondencia del Arzobispo Manuel José Mosquera con su hermano el General Tomás Cipriano (1817-1853). Bogotá: Academia Colombiana de Historia.

Mantilla, L. C. (2008). Profeta en su tierra: Hernando Arias de Ugarte V Arzobispo de Santafé de Bogotá (Su correspondencia en el Archivo General de Indias de Sevilla). Bogotá: Academia Colombiana de Historia.

Mantilla, L. C. (2013). Compartir los hallazgos. Páginas de pequeña historia de Colombia. Bogotá: Ed. Bonaventuriana.

Márquez, M. (1986). Monseñor Bernardo Herrera Restrepo en la vida politica colombiana. Bogotá: Universidad de la Sabana, Facultad de Comunicación Social y Periodismo.

Orduz, J. C. (1984). Monseñor Ismael Perdomo y su tiempo: biografía documentada del insigne Arzobispo de Bogotá. Bogotá: Antares.

Orduz, J. C. (1987). La arquidiócesis de Santafé de Bogotá en la conquista y la colonia. Bogotá: CELAM.

Osorio, J. (1938). Origen y desarrollo de la jerarquía eclesiástica en Santafé de Bogotá. Boletín de Historia y Antigüedades, 25, 485-511.

Pacheco, J. (1986). La evangelización del Nuevo Reino, siglo XVI. En Historia Extensa de Colombia Vol. XIII. Bogotá: Ediciones Lerner.

Pacheco, J. (1986). La consolidación de la Iglesia. Siglo XVII. En Historia Extensa de Colombia. Vol. XIII. Bogotá: Ediciones Lerner.

Pacheco, J. (1986). La Iglesia bajo el regalismo de los Borbones. Siglo XVIII. Libro Primero: de Felipe V a Carlos III. Libro segundo: Bajo La Ilustración. En Historia Extensa de Colombia. Vol. XIII. Bogotá: Ediciones Lerner.

Pinilla, G. (2011). La presencia de la Arquidiócesis de Bogotá en un colegio liberal: El Colegio Nacional de San Bartolomé, 1941-1951. Boletín de Historia y Antigüedades, 852, 18-32.

Restrepo, J. (1943). El primer capítulo catedral santafereño. Boletín de Historia y Antigüedades, 30, 130-152.

Restrepo, J. (1950). Arquidiócesis de Bogotá. Boletín de Historia y Antigüedades, 37, 13-28.

Restrepo, J. (1954). El capelo cardenalicio y los arzobispos de Bogotá. Boletín de Historia y Antigüedades, 41, 550-557. 
Restrepo, J. (1956). El sínodo diocesano de 1556. Boletín de Historia y Antigüedades, 43, 458-482.

Restrepo, J. (1960). El capítulo metropolitano y el 20 de julio. Boletín de Historia y Antigüedades, 47, 297-309.

Restrepo, J. (1961-1971). Arquidiócesis de Bogotá. Bogotá: Ed. Lumen Kelly, 4v.

Restrepo, J. (1967). Arzobispos y obispos de Bogotá, bogotanos que han llegado a la Mitra. Boletín de Historia y Antigüedades, 54, 363-387.

Restrepo, J. (1970). Partidas de bautismo de los arzobispos de Bogotá. Boletín de Historia y Antigüedades, 57, 87-109.

Sahamuel, E. (Dir.). (2012). Arte Sacro 450 años. Arquidiócesis de Bogotá. Bogotá: Consuelo Mendoza ediciones.

Tisnés, R. (1986). El Clero y la Independencia en Santafé, 1810-1815. En Historia Extensa de Colombia. Vol. XIII. Bogotá: Ediciones Lerner. 


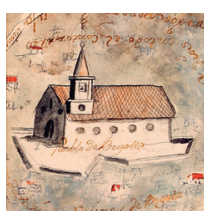

